\title{
Laboratory Scale Assessment of a Capillary Barrier using Fibre Optic Distributed Temperature Sensing (FO-DTS)
}

\author{
Robert $\mathrm{Wu}^{*}$ \\ Department of Earth and Planetary Sciences, McGill University \\ Montreal, Canada \\ *Corresponding Author \\ qiong.r.wu@mail.mcgill.ca \\ Vincent Martin \\ Department of Civil, Geological and Mining Engineering, Polytechnique Montreal \\ Montreal Canada \\ vincent.martin@polymtl.ca \\ Jeffrey McKenzie \\ Department of Earth and Planetary Sciences, McGill University \\ Montreal, Canada \\ jeffrey.mckenzie@mcgill.ca \\ Stefan Broda \\ Federal Institute for Geosciences and Natural Resources \\ Berlin, Germany \\ stefan.broda@bgr.de \\ Bruno Bussière \\ Université du Québec en Abitibi-Témiscamingue \\ Rouyn-Noranda, Canada \\ bruno.bussiere@uqat.ca \\ Michel Aubertin \\ Department of Civil, Geological and Mining Engineering, Polytechnique Montreal \\ Montreal, Canada \\ $\underline{\text { michel.aubertin@polymtl.ca }}$ \\ Barret L. Kurylyk \\ Department of Civil and Resource Engineering and Centre for Water Resources Studies - Dalhouse University \\ Halifax Canada \\ barret.kurylyk@dal.ca
}




\begin{abstract}
Recent waste rock pile designs have been proposed to incorporate a fine-grained layer to create a capillary barrier to prevent surface water from draining into the pile interior. This study analyse active fibre optic distributed temperature sensing (FO-DTS) as a tool to measure the effectiveness a capillary barrier system following an infiltration test. A laboratory waste rock column was built with anorthosite waste rock overlain by sand. Volumetric water content is calculated during heat cycles lasting $15 \mathrm{~min}$ powered at $15 \mathrm{~W} / \mathrm{m}$ in the column. A new algorithm is employed to circumvent several requirements for soil specific calibration. The inferred moisture contents were verified by soil moisture probes located adjacent to the cable.
\end{abstract}

The FO-DTS data indicate, at vertical resolutions up to $2 \mathrm{~cm}$, that water is retained in the sand and does not drain into the anorthosite following the infiltration test. The $\mathrm{R}^{2}$ between the inferred and measured volumetric water content in the fine cover sand layer is 0.90 , while the screened anorthosite maintained an $\mathrm{R}^{2}$ of 0.94 with constant moisture content throughout the test. This study will ultimately help guide future waste rock storage design initiatives incorporating fibre optic sensors, leading to improved environmental mine waste management. Keywords: DTS, Soil moisture, Distributed Temperature Sensing, Fibre Optic, Waste Rock, Capillary Barrier 


\section{Introduction}

Waste rock, extracted coarse-grained rock below the ore cut-off grade, is stored on the surface of mine sites and exposed to natural climatic conditions. The most common method to store this material is through constructed waste rock piles. These piles can be large, with heights exceeding hundreds of meters and area covering tens of hectares (Martin et al. 2005; Aubertin 2013). The typical design and construction methods for waste rock piles may create favourable conditions for the internal flow of fluids, which in turn may promote the development of acid mine drainage or contaminated neutral drainage where water is discharged as contaminated leachate from the pile (Morin et al. 1991; Bussière et al. 2005; Aubertin 2013; Chi et al. 2013). Waste rock piles generally exhibit hydrologic heterogeneity, particle segregation, sorting along slopes, and compacted layers due to machinery traffic (Bussière et al. 2003). The net-effect of these structures is the enhancement of water and air flow through the waste rock pile and the potential generation of acid mine drainage and contaminated neutral drainage (see Morin et al. 1991; Herasymuik 1996; Newman et al. 1997; Wilson et al. 2000; Aubertin et al. 2002a; Aubertin et al. 2002b; Fala et al. 2003; Fala et al. 2005; Martin et al. 2005).

Several modified designs and engineering improvements in and around waste rock piles have been proposed over the past 25 years to limit the generation of contaminated leachate. The optimal design of waste rock piles depends on their specific purpose and environmental setting. One potential design improvement is based on the concept of an inclined cover with a capillary barrier effect (CCBE) (Aubertin et al. 2002b; Bussière et al. 2003; Fala et al. 2005; Martin et al. 2005; Aubertin 2013). This type of multilayered system consists of a relatively fine-grained moisture retaining material above coarser waste rock to create a capillary break that limits infiltration into the pile interior (Rasmusson and Erikson 1986; Nicholson et al.1989; Morel-Seytoux 1992; MEND 2.22 .2 1996; Aubertin et al. 1996; Bussière and Aubertin 1999).

An effective capillary barrier exists at the interface of the coarse layer and the overlying fine material, where suction remains above the water entry value of the coarse material, preferentially retaining water in the overlay. When relatively dry, the lower coarse-grained material has a very low unsaturated hydraulic conductivity, while the fine layer exhibits stronger water retaining capabilities and tends to retain moisture (Bussière et al. 2003; Aubertin et al. 2009). When the layered system is inclined, it enhances water diversion down slope through the fine-grained layer. 
Capillary barrier systems are relatively straightforward to construct and durable, which may decrease the costs compared to other systems (Bussière et al. 2003).

To date, there are only a few technological options available for monitoring soil moisture in industrial settings. Point measurements (e.g. dielectric-permittivity soil moisture sensors) are commonly used, but they may cause difficulties during installation and when extrapolating data across large areas given system heterogeneities. Remote sensing techniques overcome some of these limitations but may resolve moisture content at resolutions that are too coarse for research purposes. In many circumstances, a combination of methods are used to understand the nature and distribution of soil moisture (e.g. Andrieau et al. 2010; Dobriyal et al. 2012).

One emerging method to evaluate soil moisture is fibre optic distributed temperature sensing (FO-DTS). This system records the thermal response of a medium to natural or engineered thermal forcing. FO-DTS can measure temperature along a fibre optic cable at fixed intervals (e.g. $0.5 \mathrm{~m}$ ) based on the temperature sensitivity of the backscattering of a light signal (Selker et al. 2003). In the last 20 years, actively heated FO-DTS techniques, for which the cable operates as both a heat source and a thermal sensor, have been developed to measure soil moisture (i.e. Weiss 2003).

The advantages of active FO-DTS is that it is a flexible sensing apparatus that can be deployed over large areas with relative ease and for indefinite periods of time. It has been shown to accurately measure temperature across a fibre optic cable over $30 \mathrm{~km}$ (Ciocca et al. 2012) and help delineate groundwater flow systems (Briggs et al. 2012). While active FO-DTS has been shown as an effective method to assess soil moisture, there are only few instances of the technology being used in applied industry settings (i.e. Weiss 2003; Benitez-Buelga et al. 2014). This technology is presently in use at an experimental waste rock pile, as part of an ongoing field monitoring program conducted by the authors and collaborators (Bréard Lanoix 2017; Martin et al. 2017).

This paper presents a laboratory experiment using active FO-DTS to measure temporal and spatial variability of aggregate and soil moisture in a large column. The main objective is to assess the applicability of active FO-DTS to resolve volumetric water (moisture) content, $\theta$, in a controlled system that encourages the formation of a capillary barrier, much like the conditions created by a CCBE. This objective is achieved by using a large experimental column for which environmental conditions are controlled. In this study, the heating profiles of a fibre optic cable are used directly to infer volumetric moisture contents rather than the common approach of integrating the heating 
profiles to infer thermal properties soil to then estimate the moisture content through an empirical calibration curve. In other words, the approach discussed in this paper circumvents the need to determine the soil specific thermal properties (i.e. heat capacity, specific heat) to estimate $\theta$ and builds upon earlier studies developing semi-empirical approaches to evaluate the moisture content (Sayde et al. 2010). This technique is beneficial when working with heterogeneous and granular (cohesionless) media where thermal properties can vary greatly with grain size distribution and grain contact with the fibre optic cable.

\section{Methods}

\section{Fibre Optic Distributed Temperature Sensing (FO-DTS)}

FO-DTS uses fibre optic technology with nonelastic Raman backscattering. In Raman backscattering, a change in the intensity of the reflected light occurs when incident light strikes the fibre optic glass wall and causes backscattering at two frequencies, which are referred to as Stokes and anti-Stokes (Selker et al. 2003). The intensity of backscatter at the anti-Stokes frequency is dependent on the temperature of the cable, thus temperature can be calculated from the ratio of the intensity of the anti-Stokes to Stokes (Grattan and Sun 2000; Ciocca et al. 2012). The precision of this measurement is proportional to the square root of the integration period or the square root of the time in one step interval assuming no errors in temperature drift (Selker et al. 2006; Sayde et al. 2010; Ciocca et al. 2012).

FO-DTS systems are portable and can be used for various environmental and hydrologic applications (e.g. Selker et al. 2006; Briggs et al. 2012; Bense et al. 2016). The rugged and compact nature of this tool makes it useful for evaluating soil characteristics in harsh conditions, such as inside a waste rock pile (Broda et al. 2013). FO-DTS systems are often jacketed with braided steel cabling, and are resistant to heavy strain and weight, but are still able to monitor rapid thermal responses in the surrounding environment.

\section{Resolving Volumetric Water Content with Heat}

The metal sheath that surrounds fibre optic cables generates heat when electrical current passes between two points on the cable. When the cable is buried in a porous medium (e.g. soil or waste rock), the change in temperature induced by the potential difference in the fibre optic cable is dependent on the thermal properties of the material surrounding the cable, including the porous material and its moisture content. If the thermal properties of the solid 
components of the porous media do not change with time during measurements, any changes in temperature when electricity is applied should be the result of changes in moisture content in the medium. As air has a very low thermal conductivity and density, it is assumed to have negligible effects on the bulk thermal properties of the medium (de Vries 1963; Kluitenberg 2002).

Several methods have been proposed to calculate the volumetric moisture content from active FO-DTS data based on classic equations in the heat diffusion literature. Carslaw and Jaeger (1959) provide an analytical solution to solve an infinite line heat source problem in a homogenous and isotropic medium using an integrated form of Fourier's law (de Vries 1963; Bristow et al. 1994; Ciocca et al. 2012; Benitez-Buelga et al. 2016). The change in temperature, $\Delta T$, in response to a constant heating strength per unit length of cable $Q(\mathrm{~W} / \mathrm{m})$, during a prolonged heating cycle (>10 minutes) can be expressed as follows (Blackwell 1954; Li et al. 2016):

$$
\Delta T(t)=\frac{Q}{4 \pi \lambda} \ln (t)+B
$$

where $Q$ is the source heating strength $(\mathrm{W} / \mathrm{m}), t$ is the heating pulse duration $(\mathrm{s}), \lambda\left(\mathrm{W} /\left(\mathrm{m}{ }^{\circ} \mathrm{C}\right)\right)$ is the thermal conductivity of the medium, and constant $B$ is a bulk parameter $\left({ }^{\circ} \mathrm{C}\right)$ that functions as the intercept when $\Delta T$ is plotted against $\ln (t)$. For active FO-DTS moisture analysis, Equation (1) is the most common solution for the single probe method in which the fibre optic cable metal sheathing acts as the infinite line source probe with known heating power and duration (Weiss, 2003). Fitting temperature data to the cooling phase after the heating cycle has been shown to also work as well for thermally conductive media (see Kluitenberg et al. 1993; Weiss 2003; Ciocca et al. 2012; Benitez-Buelga 2016).

Thermal conductivity and diffusivity can be obtained by fitting a linear regression between the temperature data and the natural logarithm of the heating duration if the integration period is long. The thermal conductivity is inferred from the slope of that regression (i.e. slope $=\mathrm{Q} /(4 \pi \lambda)$, see Equation 1) (Carslaw and Jaeger 1959; Kluitenberg et al. 1993; Bilskie 1994; Bristow et al. 1994; Weiss 2003). After obtaining $\lambda, \theta$ can be calculated using empirical models (i.e. Johansen 1975; Côté and Konrad 2005; Lu et al. 2007) or by calibrating $\theta-\lambda$ relationships with in situ probes (Sayde et al. 2010; Gil-Rodriguez et al. 2012). 
A disadvantage of this approach arises when distinguishing small changes $(<0.05)$ in volumetric water content during a short-duration heat pulse (Weiss 2003; Perzlmaier et al. 2004). Ciocca et al. (2012) obtained accurate $\theta$ values in wet media with a precision of $0.01-0.035 \mathrm{~m}^{3} / \mathrm{m}^{3}$ using a time correction applied to this method. However, to maintain a good relationship in drier media that are less thermally conductive than those considered by Ciocca et al. (2012), longer integration periods are required for the temperature profile to become asymptotic in the thermal response analysis. Dong et al. (2017) demonstrated that such method results in a weak function of soil moisture by using the cooling phase of the heating response, and that the approach may return implausible values for moisture contents if inefficient electric power is used. As such, this method may be impractical for resolving relatively rapid changes in $\theta$ due to water movement.

The dual probe heat method is an alternative approach that resolves volumetric heat capacities as opposed to thermal conductivity. The method is outlined by Campbell et al. (1991), following Carslaw and Jaeger (1959). One probe (i.e. a fibre optic cable) is uniformly heated and the thermal response is measured with a separate cable. The temperature response recorded by the unheated cable and is, in part, a function of volumetric moisture content. The temperature rise, $\Delta T$, is a function of the volumetric heat capacity of the material, $C\left(\mathrm{~J} /\left(\mathrm{m}^{3}{ }^{\circ} \mathrm{C}\right)\right)$, and the radial distance, $r(\mathrm{~m})$, between the heating source and the sensing probe (Carslaw and Jaeger 1959; Campbell et al. 1991; Bristow et al. 1994; Benitez-Buelga et al. 2014):

$$
\Delta T=\frac{q}{e \pi r^{2} C}
$$

where $q$ is the heat input per unit of length $(\mathrm{J} / \mathrm{m})$ and $e$ is Euler's constant (i.e. the base of a natural logarithm). For a porous media, $C$ is expressed as a volumetrically weighted arithmetic mean of the heat capacities of the medium constituents (de Vries 1963; Kluitenberg 2002; Benitez-Buelga et al. 2014):

$$
C=\rho_{b} c_{s}+\rho_{w} c_{w} \theta
$$


where $\rho_{b}$ and $\rho_{w}$ are respectively the bulk medium and water densities $\left(\mathrm{g} / \mathrm{m}^{3}\right)$, and $c_{s}$ and $c_{w}$ are the specific heat capacities of the solid medium and water respectively $\left(\mathrm{J} / \mathrm{kg}{ }^{\circ} \mathrm{C}\right)$. This formulation ignores the very minor influence of air and water vapor on bulk heat capacity and assumes that the pore water is entirely unfrozen.

Equations (2) and (3) can be combined to infer moisture contents from the temperature rise due to heating. However, a major disadvantage of this method is the need for very accurate probe spacing. Equation (2) shows that small changes in radial distance, $r$, can have large impact on estimations of $C$, and thereby influence $\theta$ estimates. In cases where the medium contains coarse and angular particles, the contact between the fibre optic cable and the medium and the distance between cables may shift during the construction, rendering $r$ inaccurate. Another disadvantage of this approach is that the dry bulk density and heat capacity of the medium must be known. This again can be problematic when the material is heterogeneous and coarse-grained.

Sayde et al. (2010) developed a semi-empirical approach to estimate $\theta$ using active FO-DTS. Instead of deriving thermal properties of the soil medium, the heating profile is integrated directly from FO-DTS results and extrapolated from a rating curve to fit dry and saturated conditions using an empirical model. The calculated error associated with this method is less than $0.05 \mathrm{~m}^{3} / \mathrm{m}^{3}$ in wet soil (Sayde et al. 2010). The results can be obtained using a cumulative temperature rise over time, $T_{\text {cum }},\left({ }^{\circ} \mathrm{C} \mathrm{s}\right)$, in the cable:

$$
T_{\text {cum }}=\int_{t}^{t_{0}} \Delta T d t
$$

This method assumes that the integral rise in temperature for a given heating strength is solely controlled by the bulk material properties, so the relationship between $T_{\text {cum }}$ and $\theta$ holds for a constant heating profile and fibre optic cable specification (Sayde et al. 2010; Gil-Rodriguez et al. 2012). The error associated with the changes in temperature using this method is minimized because the DTS recordings are based directly on measured temperature and do not rely on thermal properties of the material.

Zubelzu et al. (2019) compared potential (i.e. Farfan et al. 2017), exponential (i.e. Gil-Rodriguez et al. 2012), and polynomial (i.e. Benitez-Buelga et al. 2016) Tcum-moisture fitting functions. His findings suggests that all curves 
could present non-Gaussian pattern during the heating phase despite high coefficients of determination. To reduce this behaviour, the cumulative temperature profile can be integrated over a long integration period with a low sampling rate. Cable specific properties account for little of the experimental error due to the rapid increase in temperature, and these properties are obtained with a lower sampling rate during the initial temperature rise (Sayde et al. 2010; Gil-Rodriguez et al. 2012). When a higher sampling rate is used, the input amperage can be decreased so that the maximum temperature is easier to obtain. Dong et al. (2017) show that this method can yield similar results using higher power during a shorter duration and lower power over a longer integration period. This is useful where input amperage is limited in low conductive media. Striegl and Loheide (2012) provide a similar method to obtain temperature token values where the change in temperature is assumed constant and averaged over a period of 380 to 580 seconds. The token values were then related to nearby moisture sensor values to generate a temperaturemoisture curve that was calibrated to collect soil moisture data.

The relationship between $\lambda$ and $\theta$ for a given system follow a specific shape with a shallow slope at low water contents and steeper slope at higher water contents (i.e. Johansen 1975; Campbell 1991; Lu et al. 2007). The shape of the curve is a function of several specific parameters of the material. This study proposes a modified time cumulation approach to relate thermal conductivity to temperature response to obtain $\theta$. This modified approach combines the usefulness of the $T_{\text {cum }}$ approach (Equation 3 and surrounding text) with the theoretical rigor of the Blackwell (1954) approach (Equation 1). Integrating Equation (1) yields the cumulative temperature change over the heating period:

$$
T_{\text {cum }}=\frac{Q}{4 \pi \lambda} t(\ln (t)-1)+B t
$$

To account for pore and thermal variability, a Kersten Number, Ke, (Kersten 1949; Johansen 1975; Halloran 2016) is commonly used to normalize $\lambda\left(\mathrm{W} /\left(\mathrm{m}^{\circ} \mathrm{C}\right)\right)$ to soil specific dry and saturated values: 


$$
K e=\frac{\lambda-\lambda_{d r y}}{\lambda_{s a t}-\lambda_{d r y}}
$$

The degree of saturation, $S_{r}$, can then be calculated using one of many empirical models found in literature. The Lu et al. (2007) model is used here for its versatility in accurate approximations of thermal conductivity in a wide range of soils:

$$
S_{r}=\left(1-\frac{\ln (K e)}{\gamma}\right)^{\frac{1}{\gamma-1.33}}
$$

where $\gamma$ is a soil texture dependent parameter, with 0.96 and 0.27 as the values for coarse and fine textured soils respectively ( $\mathrm{Lu}$ et al. 2007). $\theta$ is then calculated from the average porosity of the material, $n$ :

$$
\theta=S_{r} \mathrm{n}
$$

\section{Experimental Setup and Measurements}

\section{Column and Helices}

The fibre optic cable was wrapped into 3 helixes held together with plastic rod scaffoldings (Figure 1). The helixes had internal radii of $0.15 \mathrm{~m}, 0.25 \mathrm{~m}$, and $0.35 \mathrm{~m}$. The cables were bent into a helical pattern to allow the FO-DTS cable to record temperature at a vertical resolution of $0.05 \mathrm{~m}, 0.03 \mathrm{~m}, 0.02 \mathrm{~m}$ in the smallest, intermediate, and largest helices respectively. The fibre optic cable passed through two cold and one warm calibration baths (Figure 2).

The helical coils were placed in a metal cylinder that is $0.80 \mathrm{~m}$ in height and has a radius of $0.80 \mathrm{~m}$. The bottom of the cylinder contained a cobble layer overlain by a geotextile to promote drainage without loss of finer particles. The 
column includes an outflow drain discharged into tipping bucket lysimeters $(1 \mathrm{~mm} /$ tip$)$ to measure effluent discharge rate. Once the cables were placed in the empty cylinder, $0.50 \mathrm{~m}$ of screened anorthosite $(<5 \mathrm{~mm})$ was poured into the column (on the geotextile) via spiral deposition to limit particle segregation and compacted layering. A homogenous sand layer $(0.20 \mathrm{~m}$ thick$)$ was then added on top of the anorthosite using the same deposition method (Figure 3). The sand had a maximum grain size of $0.5 \mathrm{~mm}$ and is considered a poorly graded fine sand (SP), while the screened anorthosite has a maximum grain size of $10 \mathrm{~mm}$ and is considered a well graded sand with silt and gravel (SW-SM) according to USCS classification (anorthosite data from Lévesque 2015).

The DTS resolves temperature to within $\pm 1.0^{\circ} \mathrm{C}$ in each material using $20 \mathrm{~s}$ dual ended measurements. To improve the accuracy of the FO-DTS measurements, data were corrected for temperature offsets and instrument drift using two reference baths with water at approximately 0 and $40{ }^{\circ} \mathrm{C}$. Van Essen Micro-Diver pressure transducers $\left( \pm 0.1^{\circ} \mathrm{C}\right.$ accuracy, $0.01{ }^{\circ} \mathrm{C}$ resolution) were placed in the baths to record the calibration temperatures. The warm bath was kept at constant temperature and well-mixed with an aquarium heater, while the cold bath contained a slush mixture. A sprinkler affixed $40 \mathrm{~cm}$ above the top of the column released $2.6 \mathrm{~L} / \mathrm{min}$ of water during an infiltration test. Care was taken to use a sprinkler nozzle that evenly irrigated the top of the column without water droplets becoming too fine (misty) and to avoid droplets from adhering to and flowing down the rain jacket surrounding the column. Heterogeneity in spatial results within the column may nonetheless be caused by uneven irrigation (see results section).

\section{Instrumentation}

The FO-DTS instrument used in this experiment is an AP Sensing N4388A GeoDTS with a manufacturer spatial resolution of $1.0 \mathrm{~m}$ (vertical resolution is higher due to the helixes), sampling interval of $0.5 \mathrm{~m}$, and a minimum data integration time of $20 \mathrm{~s}$ in dual ended measurements. The fibre optic cable is the Brugg BRUsteel LLK-BST 2FG5, which has two fibre optic strands encased within a galvanized steel tube and wrapped with braided steel wire and a waterproof nylon jacket.

Four Decagon EC-5 soil moisture sensors were buried in the sand layer, at $5 \mathrm{~cm}$ and $15 \mathrm{~cm}$ depth, and four were buried in the anorthosite waste rock layer, $30 \mathrm{~cm}$ and $50 \mathrm{~cm}$ depth, to compare $\theta$ results to the DTS and parameter $\gamma$ values to Lu et al. (2007) The EC-5 sensors have a manufacturer's stated accuracy of $\pm 0.03 \theta$ in mineral soil (Decagon 2015). The sensor measures the dielectric constant of the media using the capacitance and frequency of 
the soil domain. The dielectric permittivity is utilized to determine the moisture content with the Topp Equation (Topp et al. 1980):

$$
\theta=\frac{1}{\left(-3.3325 * 10^{-9}\right)\left(m V^{3}\right)+\left(7.0218 * 10^{-6}\right)\left(m V^{2}\right)-\left(5.11647 * 10^{-3}\right)(m v)+1.30746}
$$

where $\mathrm{mV}$ is the sensor output after $2,500 \mathrm{mV}$ excitation.

The probes utilized in the experiment were individually calibrated with material specific calibration curves. The experiment sensors were calibrated using samples from the column and yielded an accuracy of $\pm 0.06 \theta$ in the sand and crushed anorthosite, which revealed that the accuracy of the probes were lower than the manufacturer's stated error. Although the crushed anorthosite is texturally more complex, the EC-5 accuracy in this material was the same as homogenous sand. This suggests that $\pm 0.06 \theta$ may be the highest accuracy achievable with the probes used in the experiment.

\section{Calibration and Determination of Parameters}

Endmembers of $T_{\text {Cum }}$ were measured independently of the infiltration test. Dry $T_{C u m}$ values were obtained before water was added into the column. Dry measurements were taken for 10 cycles to ensure the power supply provided consistent heating under dry conditions. Consecutive measurements were repeated after the infiltration test, at saturation, to ensure the power was sufficient to allow the DTS to record changes in temperature when wet. The material in the column was saturated upwards with a Mariotte bottle to ensure most of the voids were filled with water. Near-saturation conditions were expected using a low hydraulic gradient to ensure that the upward movement of finer particles had negligible influence on homogeneity.

The EC-5 soil moisture sensors essentially measure porosity under saturated conditions, assuming all pores are filled with water. The volumetric water content at saturation measured by the EC-5's was 0.44 and 0.26 respectively for the sand and anorthosite. Porosity was calculated from column samples and yielded porosity of 0.42 and 0.27 respectively for the sand and anorthosite. The obtained $\gamma$ was 0.26 and 0.92 for the cover sand and (screened) anorthosite respectively, which is close to the suggested values provided by Lu et al. (2007). The $B$ term in Equation (5) is calculated as the intercept in each heating cycle between $\Delta T$ and $\ln (t)$. 


\section{Heated Fibre Optic Measurements}

Figure 4 presents the vertical temperature profile obtained during a typical heating cycle for a dual-ended FO-DTS configuration, in which the end of the fibre optic cable returns to the DTS and measurements are collected through both ends of this cable. Dual ended measurements integrate temperature readings averaged from both ends of the cable, decreasing the chance for signal loss in longer fibre optic cables.

A heating cycle is one hour long; for the first 15 minutes the cable was actively heated by applying an electrical current across the cable followed by 45 minutes of no heating during which time the system cools. A DC power supply with a voltage regulator to maintain $15 \mathrm{~W} / \mathrm{m}$ was used throughout the heating cycles and adapted to changing resistance in the cable due to heating. The resistance of the cable was $17 \Omega$ at room temperature, and the length of heated cable was $46 \mathrm{~m}$. The power used in this experiment is higher than in previous published studies (Sayde et al. 2010; Striegl and Loheide 2012; Benitez-Buelga et al. 2016) because the temperature response in the low thermally conductive screened anorthosite was not sufficient at lower electrical power. Higher power settings also enabled measurement of smaller changes at low volumetric soil moisture levels $(<0.06 \%)$ as the uncertainty in the $\theta$ error analysis is inversely proportional to the value of $\theta$ (Weiss 2003).

\section{Results}

Fifteen-minute heating cycles were performed every hour (Figure 4a) for 190 cycles or 7.9 days. The change in temperature during the first cycle is shown in Figure $\mathbf{4 b}$. In dry conditions, the sand reached a $\Delta T$ of $35^{\circ} \mathrm{C}$ during heating, while the anorthosite had a $\Delta T$ of $20^{\circ} \mathrm{C}$. The sprinkler was turned on at the start of the $4^{\text {th }}$ cycle for 15 minutes to apply water to the experimental column Thirty-nine liters of water, equivalent to $19.4 \mathrm{~mm}$ of rain across 15 minutes to simulate a short, but intense precipitation event. Within one hour after the onset of infiltration, the temperature of the sand layer decreased by $21^{\circ} \mathrm{C}\left(\Delta T\right.$ decrease from $35^{\circ} \mathrm{C}$ to $\left.14^{\circ} \mathrm{C}\right)$ during the heating phase

(Figure 4c). The anorthosite unit did not exhibit a change in temperature, maintaining a temperature of $45^{\circ} \mathrm{C}$ during the infiltration period. When the sand and waste rock were highly saturated, the $\Delta T$ of both materials was reduced to $10{ }^{\circ} \mathrm{C}$, Figure $4 d$.

The relationship between $T_{\text {cum }}$ and $\theta$ assumes that the overall density and texture of the material remain constant. Using the relationship defined by $\mathrm{Lu}$ (2007) with specific parameters obtained here (see Experimental Setup and 
Measurements section), the variation of $\theta$ with depth within the column was calculated. Figure 5 shows one week of results after a 15 -minute wetting test (equivalent to $19.4 \mathrm{~mm}$ of rain). The test starts with three heating cycles prior to wetting to establish baseline conditions, and the infiltration event occurs during the fourth heating cycle.

Volumetric water content increases in the cover sand layer during the fourth cycle in the top $10 \mathrm{~cm}$ of the column. During infiltration, the highest $\theta$ in the sand was obtained in the interior helix and the lowest $\theta$ was in the exterior helix. Once the infiltration event ended, the exterior helix recorded the highest moisture content value postinfiltration in the sand $\left(0.42 \mathrm{~m}^{3} / \mathrm{m}^{3}\right)$, while the interior recorded the lowest $\left(0.36 \mathrm{~m}^{3} / \mathrm{m}^{3}\right)$. The highest volumetric water content was measured in the lower section of the sand layer following the infiltration test, as expected in a capillary barrier system. There was no corresponding increase in moisture in the anorthosite layer (Figure 5).

Over the course of the week, the value of $\theta$ in the upper sand layer decreased slowly, while the $\theta$ remained effectively unchanged in the anorthosite unit. By the end of the test, the $\theta$ near the interior helix in the upper sand layer approached antecedent residual $\theta$ values $\left(0.09 \mathrm{~m}^{3} / \mathrm{m}^{3}\right)$. No discharge was recorded from the column's outflow drain over the course of the experiment.

The coefficient of determination, $\mathrm{R}^{2}$, between the active FO-DTS measurements and the EC-5 soil moisture probes in the cover sand layer is 0.90 , with a root mean square error (RMSE) of 0.06 . The DTS-based measurements are more sensitive to small changes in $\theta$ than the four EC-5 soil moisture probes in the sand. For example, the comparison between the DTS and probe values at around $\theta=0.20$ is poor as changes in DTS readings do not correlate to changes recorded by the moisture probe (Figure 6). Higher $\theta$ values tend to produce larger variations as the uncertainty in the measurements are proportional to the degree of saturation.

The EC-5 moisture probes recorded $\theta$ increases in water content in less than five minutes after the onset of the infiltration event. Quick increases in $\theta$ values could not be recorded during the early part of the 15 -minute infiltration test, so no $\theta$ values in the $0.05-0.20$ range were observed in the sand layer. The error associated with calculating $\theta$ should increase with $\theta$. However, results obtained here indicate that the largest uncertainty exists around 0.20 for this infiltration test (Figure 6).

Comparison of volumetric water content values during the infiltration test measured by the active FO-DTS and moisture probes yielded a value of approximately 0.15 and a mean absolute error (MAE) of 0.07 in the anorthosite waste rock. Using both the $\theta$ values during the infiltration test and during saturation, a $\mathrm{R}^{2}$ of 0.94 was obtained. It 
was difficult to assess the accuracy of the active FO-DTS moisture data in the anorthosite layer because there was only a small range of $\theta$ values (e.g. $0.10-0.18$ ). During the entirety of the infiltration test, the value of $\theta$ in the anorthosite layer remained relatively constant (Figure 5), so only dry, slightly wet, and saturated values were obtained for that layer.

\section{Discussion}

The results obtained from the FO-DTS suggest that water was confined to the fine sand layer which may have been caused by inherent water retention (capillary) capabilities between the sand and the anorthosite (Figures 4 and 5). The post infiltration water content in the sand did not reduce suction, at the interface between the layers, below the water entry value of the anorthosite layer. Thirty-nine liters of water was not sufficient to induce drainage into the anorthosite layer in an estimated $47 \mathrm{~L}$ void space. More water was not added due to observed ponding on the top of the sand layer at the given infiltration rate. In earlier tests, $52 \mathrm{~L}$ and $73 \mathrm{~L}$ of water was applied to the column in 20 and 30 minutes respectively. These tests were disregarded as there was water ponding on the surface of the cover sand unit which flowed out towards the edge of the column. However, the DTS resolved water flowing through the interface from the sand and into the anorthosite. In both cases, water discharged from the outflow drain approximately 35 minutes after the start of infiltration, but the outflow quantity was too much for the tipping bucket rain gauge to record.

The difference in $\Delta T$ of the anorthosite between dry and saturated conditions was only 5 to $10^{\circ} \mathrm{C}$. This suggests using a high-power output was warranted as less power may not have resolved the difference in dry and saturated conditions in this layer. Figure 7 shows a 2D integration of temperature using all three helices; see supplementary material S2 and S3 to see temperature changes during the infiltration test. Spatial and temporal temperature differences in the sand and anorthosite are easily distinguished; however, it is difficult to quantify the impact of these factors on the irregularities in this figure. Note that the laboratory environment was humid; a drier environment may have produced more pronounce decreases of $\theta$ in the upper sand.

The results show that much of water appears to enter the near the center of the column during the start of the infiltration test (Figure 5). Water then migrates towards the exterior of column after the infiltration test as water content was highest in the outer helix. The outer helix had the smallest vertical resolution which resolved finer vertical changes in $\theta$. The unevenness in temperature in the sand layer may have been due to physical phenomenon 
such as local compaction of the material and the amount of contact between the cable and the medium. It could also have been due to interpretive methods when designing the helical data in 2D space. Decisions such as the type of interpolation used (i.e. linear, spline, cubic) for the figure and how the helical path is represented in $2 \mathrm{D}$ affects each respective helix (i.e. sine, cosine). The heated profile was not uniform throughout the column. Bends in the helical structure cause changes in DTS intensity ratio, and limitations in temperature resolution due to molecular vibrations causing broadening of Raman peaks, instrument noise due to transmission loss, and local moisture pockets. Sand particulates may have moved into the pore spaces of the anorthosite layer during the compaction and infiltration process. It is likely that the uneven results were caused by a combination of these factors.

The anorthosite layer was not completely dry before the onset of the week-long infiltration test due to conditions from previous experiments. Figure 5 shows pronounced horizontal layering in the underlying anorthosite unit (see between depths 22 and $72 \mathrm{~cm}$ ). This distinctive pattern may be due to density differences in the anorthosite from layering during the construction phase. It could also be due to small unwarranted bends in the helical structure causing interpretive errors during measurement. The spatial resolution of the DTS system is $1 \mathrm{~m}$; a system with a higher resolution might be able to better identify if this patterning is from bedding or instrument artefact.

Volumetric water content in the anorthosite remained constant throughout the experiment, but not across the helical path (Figure 5). This character may be produced by preferential flow paths that carried finer particles in clumps and formed layers resistant to pore water flow. However, irregularities in the helical path and differences in bulk densities could also contribute to the source of error. The column was filled by means of spiral deposition to minimize the degree of horizontal layering, but given the dimensions of the experiment, such irregularities are inevitable. Although the anorthosite is coarser than the sand, it compared more favourably with the EC-5 sensors than the sand.

Increasing power applied to the cable was effective in reducing error of the measurements (Sayde et al. 2012; Dong et al. 2017). However, although the anorthosite had a much lower thermally conductivity and thus temperature change $\left(\Delta T\right.$ of $20^{\circ} \mathrm{C}$ when dry) than the sand $\left(\Delta T\right.$ of $35^{\circ} \mathrm{C}$ when dry), it maintained accurate results (Figure 6). All three helices exhibited similar temperature and moisture values where the largest helix had the highest spatial resolution (Figure 5). However, given the proximity of the outer helix to the metal wall of the column, there may have been interference to the thermal response. The possibility of heat escaping through the sides of the metal 
enclosure or ambient air affecting readings near the column wall were not significant sources of error for the following reasons:

(1) Ambient temperatures near the helices were all within $1.0^{\circ} \mathrm{C}$ of each other, indicating that there was not significant temperature difference between the column wall and center.

(2) Three inches of fibreglass insulation was wrapped around the outer wall of the column to isolate the soil from the outside environment.

(3) The process to calculate $T_{\text {cum }}$ does not change if the ambient air and soil temperatures remain above $0{ }^{\circ} \mathrm{C}$.

Like previous studies (e.g. Sayde et al. 2010; Ciocca et al. 2012; Gil-Rodríguez et al. 2012; Striegl and Loheide 2012; Dong et al. 2017), the error associated with measuring $\theta$ increases with $\theta$ magnitude (Figure 8). The relationship between $T_{\text {cum }}$ and $\theta$ follows an exponential function (Equation 7) where small changes in $T_{\text {cum }}$ at high $\theta$ affects results more than at low $\theta$. A potential source of error may be overheating because the uncertainty along the saturated profile is greater than the dry profile (Figure 8). However, if an excess of power is the cause of this error, the result should be proportional to its magnitude, where the error associated with $\theta$ should appear largest during dry conditions. Given the mean absolute error of the DTS compared to the EC-5 sensors (RMSE of 0.06 in the sand unit and MAE of 0.07 in the anorthosite unit), the agreement of the results to moisture probes is good. Since the moisture sensors measured $\theta$ at a single location, the comparison between the sensors and the DTS only exists in 8 places within the column (4 in the sand and 4 in the anorthosite layer). The DTS system also has a large spatial average (1 m) which integrates temperature readings from a very large section of soil versus the EC-5's precise measurement area.

Note the EC-5 sensors were not highly accurate for the materials used in the column. In an independent test, two EC-5 sensors (that were used in this experiment) were placed into a small container, with $10 \mathrm{~cm}$ spacing. The container was filled with screened anorthosite. The two sensors differ in readings by $0.07 \theta$ under dry conditions in anorthosite and $0.05 \theta$ in wet conditions; no calibration was imparted on these values for the test. This test provides an assessment of the potential error in sensor measurements. Dong et al. (2017) investigated the relationship between EC-5 readings to measurement depth and orientation. Their study concludes a bias in the EC-5 sensors regarding proximity and orientation to the surface of the medium against DTS readings. The difference in 
measurement volume between the EC-5 and DTS can also be attributed to contact resistance to the medium which could allow air to act as an isolating layer that exists in the soil's macropores.

In a water balance exercise for the infiltration period, $39 \mathrm{~L}$ of water was estimated to have been infiltrated into the sand layer of the column. The volume of water added during the infiltration test estimated by spatially integrating values obtained by the 3 helices is $32.6 \mathrm{~L}$. Thus, the FO-DTS method underestimates the amount of infiltrated water by $16 \%$ in the column. The capacity of the sand to hold the water, assuming homogenous porosity and no change in pore fraction due to infiltration, is $47.3 \mathrm{~L}$. There are methodological variables that may explain the difference between the infiltration volume and measured soil moisture in the sand layer. The measurement of infiltration water may be overestimated. Small amounts of water may have infiltrated into the anorthosite, although not enough to be observed with the DTS. Furthermore, some water may have flowed preferentially along the column walls or in macropores (without being detected). The interior helix in Figure 5 has low vertical resolution and showed that water may have penetrated the sand-anorthosite interface, but it is difficult to quantify the actual impact of the lowresolution reading and seepage.

With each consecutive hourly heating cycle (15 minutes of active heating followed by 45 minute of cooling), the ambient temperature within the column increased by nearly $1{ }^{\circ} \mathrm{C}$. However, the increase in temperature during each heating phase remained constant. Weiss (2003) and Sayde et al. (2010) showed that heating cycles do not affect soil compaction. Similarly, our experiment showed no changes in $T_{\text {cum }}$ between cycles indicating that there were not any significant distortions affecting the soil due to cumulative heating effects.

The purpose of using a long integration period is to decrease error of the initial temperature rise in case it is influenced by characteristics of the cabling (a concern for thicker cables) rather than the soil medium. There are few studies detailing the relationship between hollow gaged wires and resistive heating under electrical load. In past studies, researchers did not integrate the initial phase in case the cable material was a unique variable in the integration step (Striegl and Loheide 2012; Broda et al. 2013). A long integration period reduces this error of the initial temperature rise as it becomes only a small part of total temperature integration period.

Researchers have noted that using an asymptotic relationship during heating cycles offers the advantage of not needing to account for the effects of the confining fibre optic cable material if the heating cycle is long enough (Ciocca et al. 2012). Our tests show that longer integration times are needed for low conductivity materials (i.e. 
crushed anorthosite). However, longer integration periods may mask quicker changes in water content that could take place in short periods of time. With our $\Delta T$ and power settings, the impact of a long integration period using a time cumulating approach also does not seem to be due to instrument fatigue; however, this should be considered on an individual case basis. The temperature of the fibre optic cable in the experimental medium should be within manufacturer's guidelines, and one should also ensure the temperature at the fibre optic and heating cable connection is also within limits. Temperature readings at the connection may not be accurate, so the data should not be integrated from within the spatial averaging distance of the connection.

Several other models also follow this trend when relating $\lambda$ and $\theta$ (e.g. Johansen (1975); Farouki (1981); Chung and Horton (1987); Cote and Konrad (2005), Sayde et al. (2014); and Barry-Macaulay et al. (2015)). The Lu et al. (2007) model relates $\lambda$ and $\theta$ for a wide range of soils, this technique is here considered to be the most appropriate for screened anorthosite as the majority of other models targeted for quartz sand or loam layers. With the calibrated $\gamma$ parameters, the methods presented in this study yielded $\theta$ with errors of 0.06 in the sand and 0.07 in the anorthosite. Although earlier methods have obtained more precise moisture estimations in different materials (see He et al. (2018) for a record of moisture estimations using DTS), the purpose of this paper is to highlight the potential to use DTS as a standalone measurement tool without using an empirically calibrated soil moisture response curve.

\section{Reccomendations and Conclusion}

By using active FO-DTS method to measure soil moisture, the authors were able to vertically delineate $\theta$ during an infiltration test. Over the course of one week, the feasibility of using a fibre optic helical network to observe a system that favors the formation of a capillary barrier was tested. This is the first study that does not use an empirically derived calibration curve to obtain soil specific moisture contents. This is ideal for coarse and heterogenous material where thermal properties are not easily derived. This active FO-DTS method utilizes the relationships between $T_{C u m}$ and $K e$ and between $K e$ and $\theta$ in the Lu et al. (2007) model of saturation. Using $T_{C u m}$ offers advantages over other methods that involve thermal properties of the medium, because the precision of this calculation is solely a function of temperature measured from the DTS and does not rely on inferring properties for site-specific soils. All soils follow the same general shape regarding time integration of temperature, $T_{C u m}$, and $\theta$ so site-specific calibration curves can be adjusted from this general model. Comparison of $\theta$ is completed with 8 EC-5 
probes with satisfactory agreement $-\mathrm{R}^{2}$ value of 0.90 in the sand and 0.94 in the anorthosite. One-hour measurements indicate that the sand layer could retain and hold $39 \mathrm{~L}$ of infiltration (at $2.6 \mathrm{~L} / \mathrm{min}$ ). The FO-DTS measured a slow drying/evaporation event following the infiltration event in each of the helices.

The results presented here demonstrate that FO-DTS can continuously resolve changes in $\theta$, even across fine boundaries created by a capillary break. Calibrating active FO-DTS data through $T_{\text {cum }}$ presents some challenges even in laboratory conditions as methods rely on models that fit data to characteristic shapes based on behavior of a selection of soil types. A prominent difficulty in using this method for consecutive cycles comes from the reliability of the power source. The resistance, and thus power requirement, of a metal wire depend on the temperature of the wire. It is thus necessary that the power supply be maintained regardless of temperature, especially if the experiment will be duplicated in field conditions where the ambient temperature can change diurnally and seasonally. Balancing the right amount of power to the soil properties is a challenge. This challenge was overcome by applying sufficient power to enable the lowest conductivity material (crushed anorthosite) to maintain a constant $\Delta T$ of $10^{\circ} \mathrm{C}$. However, this approach was compromised where such power made it difficult for the sand unit to return to its initial ambient temperature following several consecutive heating cycles. Each succeeding heating cycle raised the ambient temperature in the sand by nearly $1{ }^{\circ} \mathrm{C}$. Also, due to the long heating cycles, the methods presented herein were not able to resolve rapid changes in $\theta$ in the sand layer as water migrated to the bottom of the sand within 5 minutes from the onset of infiltration. Future experiments in thermally conductive materials should use shorter heating periods with a higher sampling rate. However, quicker sampling rates decrease with accuracy of the measurement, so integration time and sampling rate should be managed based on the DTS system specification.

Longer tests would also need to account for hysteresis caused by repeated heating and drying cycles. This phenomenon along with soil healing adds to complexity during cable installation. Vibratory compaction methods can cause lateral soil displacement and create low hydraulic conductivity zones (Sourbeer and Loheide 2015). This can be problematic where heavy machinery is commonly used during the construction process for large active DTS experiments. New methods are needed to estimate contact resistance and account for long term soil moisture calculations in fine materials.

The resistance of the fibre optic cable is an important factor when creating a DTS protocol. Low resistance cables can be difficult to heat due to their low electrical resistance. On the other hand, resistive cables require more voltage 
to generate and sustain power. Generating constant voltage is critical and a voltage and power regulator may be needed to account for changes in the resistance of the cable during heating cycles. Deciding on the type of fibre optic cable may be difficult if the thermal properties of the medium are not known. Further assessment will be required to understand how hysteresis will affect soil conditions during repeated cycles in field conditions where diurnal and seasonal changes can influence results. A future endeavor for this method is to detect water flux in transient environments. Since $T_{\text {cum }}$ is a function of flow around DTS cables, a high density DTS network could be able to detect rapid changes in high intensity environments.

This study encourages future field work where FO-DTS can be used as a new tool to observe the internal hydrogeology of potential capillary action, for example CCBE waste rock pile covers. The helical design is ideal for laboratory scale experiments where vertical resolution is more important than horizontal, however this may not the case for large field experiments. The most important locations to measure moisture in a water retaining portion of the waste rock pile is immediately above and below the cover. Waste rock piles have a flat rectangular top; thus, an ideal shape to install a fibre optic system would be in a grid pattern for low powered tests, or stripe-like pattern for high powered tests. These patterns would ideally be placed above and below the capillary barrier as one single, continuous cable, although two cables may be used if the resistance of the cable is too high due to the length of cable (Martin et al. 2017).

Independent soil moisture values must be taken at the field site if the compaction of the soil differs from laboratory settings. Site specific $T_{\text {cum }}$ values at dry and saturated conditions may be required as well if the material is not homogenous and well-graded. Saturated values could be taken after a large infiltration event in the field, while dry values can be taken in a controlled area possibly close to the surface of the waste rock pile. This method is also limited to temperatures above $0{ }^{\circ} \mathrm{C}$ as below freezing conditions could create complications regarding melting ice and the latent heat effect.

This experiment preceded a meso-scale test on an experimental waste rock pile. The engineered structure was designed based on numerical simulations to assess control approaches to water drainage in waste rock piles (see Martin et al. 2017). Knowledge of FO-DTS behavior from this experiment will be transferred to this large field project. More than $1 \mathrm{~km}$ of fibre optic cable was installed in the cover, waste, and bottom layer of the pile to observe the efficiency of the flow control layer (FCL). This FCL was inclined at 5 degrees to promote waterflow downslope 
to the toe of the pile where is expected to drain. FO-DTS provides insight on the performance of the top FCL by providing information on the water content in the pile to observe if water is diverted to the toe or if it infiltrates into the waste rock.

\section{Acknowledgements}

The authors would like to thank the Research Institute on Mines and Environment (RIME) for their support. Special thanks to Lukas Cheung (McGill University), Noura El-Harrak (Polytechnique Montréal), and Thomas Pabst (Polytechnique Montréal) for their invaluable contributions to this project. This work was funded by the Natural Sciences and Engineering Research Council of Canada (NSERC), Rio Tinto, and partners of RIME.

\section{References}

Anterrieu, O., Chouteau, M., and Aubertin, M. 2010. Geophysical characterization of the large-scale internal structure of a waste rock pile from a hard rock mine. Bulletin of Engineering Geology and the Environment,69(4), 533-548. doi:10.1007/s10064-010-0264-4.

Aubertin, M., Bussière, B., Aachib, M., Chapuis, R. P., and Crespo, J. R. 1996. Une modélisation numérique des écoulements non saturés dans des couvertures multicouches en sols. Hydrogéologie. 1:3-13.

Aubertin, M., Bussière, B., and Bernier, L. 2002a. Environnement et gestion des rejets miniers [CD-ROM] Montréal, Québec, Les Presses Internationales de Polytechnique.

Aubertin, M., Fala, O., Bussière, B., Martin, V., Campos, D., Gamache-Rochette, A., Chouteau, M., and Chapuis, R.P. 2002b. Analyse des écoulements de l'eau en conditions non saturées dans les haldes à stériles, in Défis et perspectives: Symposium 2002 sur l'Environnement et les Mines, CIM, [CD-ROM] Rouyn-Noranda, Canada.

Aubertin, M., Cifuentes, E., Apithy, S. A., Bussière, B., Molson, J., and Chapuis, R. P. 2009. Analyses of water diversion along inclined covers with capillary barrier effects. Canadian Geotechnical Journal, 46(10), 11461164. doi:10.1139/t09-050.

Aubertin, M. 2013. Waste rock disposal to improve the geotechnical and geochemical stability of piles. In Proceedings of the world mining congress, Montreal, Canada.

Barry-Macaulay, D., Bouazza, A., Wang, B., and Singh, R. M. 2015. Evaluation of soil thermal conductivity models. Canadian Geotechnical Journal, 52(11), 1892-1900.

Bense, V. F., Read, T., Bour, O., Borgne, T. L., Coleman, T., Krause, S., and Selker, J. S. 2016. Distributed Temperature Sensing as a downhole tool in hydrogeology. Water Resources Research, 52(12), 9259-9273. doi:10.1002/2016wr018869.

Benítez-Buelga, J., Sayde, C., Rodríguez-Sinobas, L., and Selker, J. S. 2014. Heated Fibre Optic Distributed Temperature Sensing: A Dual-Probe Heat-Pulse Approach. Vadose Zone Journal, 13(11).

Bilskie, J. R. 1994. Dual probe methods for determining soil thermal properties: Numerical and laboratory study.

Blackwell, J. H. 1954. A Transient-Flow Method for Determination of Thermal Constants of Insulating Materials in Bulk Part I-Theory. Journal of Applied Physics, 25(2), 137-144. doi:10.1063/1.1721592.

Bonan, G. B. 2008. Forests and Climate Change: Forcings, Feedbacks, and the Climate Benefits of Forests. Science, 320(5882), 1444-1449. doi:10.1126/science.1155121z. 
Bristow, K. L., Kluitenberg, G. J., and Horton R. 1994. Measurement of soil thermal properties with a dual-probe heat-pulse technique. Soil Sci. Soc. Am. J. 58:1288-1294.

Briggs, M. A., Lautz, L. K., and McKenzie, J. M. 2012. A comparison of fibre-optic distributed temperature sensing to traditional methods of evaluating groundwater inflow to streams. Hydrological Processes, 26(9), 1277-1290.

Bréard Lanoix, M.-L. 2017. Caractérisation des propriétés hydrogéologiques de la couche de contrôle des écoulements placée sur la halde à stériles expérimentale à la mine du lac Tio (Masters thesis, École Polytechnique de Montréal).

Broda, S., Aubertin, M., Blessent, D., Hirthe, E., and Graf, T. 2014. Improving control of contamination from waste rock piles. Environmental Geotechnics, 4(4), 274-283.

Broda, S., Sayde, C., Selker, J. S., Aubertin, M., and Blessent D. 2013, Using temperature as a tracer for analyzing the response of a capillary barrier in waste rock, paper presented at GeoMontreal 2013: Geosciences for Sustainability, 66th CGS Conference, Montreal, Que.

Bussière, B., and Aubertin, M. 1999. Clean tailings as cover material for preventing acid mine drainage: an in situ experiment. In Proceedings of Sudbury '99, Mining and the Environment, Sudbury, Ont. 13-17 September 1999. Vol. 2, pp. 19-28.

Bussière, B., Aubertin, M., and Chapuis, R. P. 2003. The behavior of inclined covers used as oxygen barriers. Canadian Geotechnical Journal, 40(3), 512-535. doi:10.1139/t03-001.

Bussière, B., Aubertin, M., Zagury. G. J., Potvin. R., and Benzaazoua, M. 2005. Principaux défis et pistes de solution pour la restauration des aires d'entreposage de rejets miniers abandonnées. Symposium 2005 sur l'environnement et les mines.

Campbell, G. S., Calissendorff, C., and Williams, J. H. 1991. Probe for Measuring Soil Specific Heat Using A HeatPulse Method. Soil Science Society of America Journal. 55. 291-293.

Carslaw, H. S., and Jaeger, J. C. 1959. Conduction of heat in solids. Oxford: Clarendon Press.

Chi, X., Amos, R. T., Stastna, M., Blowes, D. W., Sego, D. C., and Smith, L. 2013. The Diavik Waste Rock Project: Implications of wind-induced gas transport. Applied Geochemistry, 36, 246-255. doi: 10.1016/j.apgeochem.2012.10.015.

Chung, S.-O., and Horton, R. 1987. Soil heat and water flow with a partial surface mulch, Water Resources. Res. 23(12), 2175-2186.

Ciocca, F., Lunati, I., Giesen, N. V., and Parlange, M. B. 2012. Heated fibre optic for Distributed Soil-Moisture Measurements: A Lysimeter Experiment. Vadose Zone Journal, 11(4). doi:10.2136/vzj2011.0199.

Côté, J., and Konrad, J.M. 2005. A generalized thermal conductivity model for soils and construction materials. Canadian Geotechnical Journal. 42. 443-458. 10.1139/t04-106.

Decagon, 2015. EC-5 Volumetric Water Content Sensor: Manual. http://manuals.decagon.com/Manuals/13876_EC5_Web.pdf

de Vries D.A. 1963. Thermal properties of soil. In: Physics of Plant Environment (ed. W.R. van Wijk) NorthHolland, Amsterdam, 210-235, 1963.

Dobriyal, P., Qureshi, A., Badola, R., and Hussain, S. A. 2012. A review of the methods available for estimating soil moisture and its implications for water resource management. Journal of Hydrology, 458, 110-117.

Dong, J., Agliata, R., Steele-Dunne, S., Hoes, O., Bogaard, T., Greco, R., and van de Giesen, N. 2017. The Impacts of Heating Strategy on Soil Moisture Estimation Using Actively Heated Fibre Optics. Sensors (Basel, Switzerland), 17(9), 2102. http://doi.org/10.3390/s17092102.

Fala, O., Aubertin, M., Molson, J. W., Bussiere, B., Wilson, G. W., Chapuis, R., and Martin, V. 2003. Numerical modelling of unsaturated flow in uniform and heterogeneous waste rock piles. In Sixth International Conference 
on Acid Rock Drainage (ICARD), Australasian Institute of Mining and Metallurgy, Cairns, Australia, Publication Series (Vol. 3, pp. 895-902).

Fala, O., Molson, J., Aubertin, M., and Bussière, B. 2005. Numerical modelling of flow and capillary barrier effects in unsaturated waste rock piles. Mine Water and the Environment, 24(4), 172-185.

Farfan, J. L. S., Muñoz, J. F., and Suárez, F. 2017. Assessment of the active method to determine soil moisture. Ingeniería del Agua, 21(3), 165-178.

Gil-Rodríguez, M., Rodríguez-Sinobas, L., Benítez-Buelga, J., and Sánchez-Calvo, R. 2013. Application of active heat pulse method with fibre optic temperature sensing for estimation of wetting bulbs and water distribution in drip emitters. Agricultural Water Management, 120, 72-78. doi: 10.1016/j.agwat.2012.10.012.

Grattan, K. T. V., and Sun, T. 2000. Fibre optic sensor technology: An overview. Sensors and Actuators, A: Physical, 82(1), 40-61. doi:10.1016/S0924-4247(99)00368-4.

Halloran, L. J., Rau, G. C., and Andersen, M. S. 2016. Heat as a tracer to quantify processes and properties in the vadose zone: A review. Earth-Science Reviews, 159, 358-373. doi:10.1016/j.earscirev.2016.06.009.

He, H., Dyck, M. F., Horton, R., Li, M., Jin, H., and Si, B. 2018. Distributed Temperature Sensing for Soil Physical Measurements and Its Similarity to Heat Pulse Method. Advances in Agronomy, 173-230. doi:10.1016/bs.agron.2017.11.003

Herasymuik, G.M. 1996. Hydrogeology of a sulfide waste rock dump, Master's Thesis in Civil Engineering, University of Saskatchewan, $184 \mathrm{pp}$.

Johansen, O. 1975. Thermal conductivity of soils. Ph.D. thesis. Trondheim, Norway (CRREL Draft Translation 637, 1977) ADA 044002.

Kersten, M.S. 1949. Thermal properties of soils. Bulletin 28. Minneapolis: Engineering Experiment Station, University of Minnesota.

Kluitenberg, G. J., Ham, J. M., and Bristow, K. L. 1993. Error Analysis of the Heat Pulse Method for Measuring Soil Volumetric Heat Capacity. Soil Science Society of America Journal. Soil Science Society of America, 57(6), 1444.

Kluitenber, G. J. 2002. Thermal Conductivity. In: J.H. Dane and G.C. Topp, editors, Methods of soil analysis. Part 4. Physical methods. SSSA Book Ser. 5. SSSA, Madison WI. P. 1201-1209.

Lévesque, I. 2015. Caractérisation hydrogéotechnique des roches stériles tamisées et compactées à l'aide d'essais de laboratoire à une échelle intermédiaire. Masters' thesis, Polytechnique, Montreal, QC, Canada.

Li, M., Si, B. C., Hu, W., and Dyck, M. 2016. Single-Probe Heat Pulse Method for Soil Water Content Determination: Comparison of Methods. Vadose Zone Journal,15(7), 0. doi:10.2136/vzj2016.01.0004.

Lu, S., Ren, T., Gong, Y., and Horton, R. 2007. An Improved Model for Predicting Soil Thermal Conductivity from Water Content at Room Temperature. Soil Science Society of America Journal,71(1), 8. doi:10.2136/sssaj2006.0041.

Martin V., Aubertin, M., Zhan, J., Bussière, B., and Chapuis, R.P. 2005. An investigation into the hydrological behaviour of exposed and covered waste rock dumps. Society for Mining, Metallurgy and Exploration (SME) Transactions 318: 139-146.

Martin, V., Bussière, B., Plante, B., Pabst, T., Aubertin, M., Medina, F., Bréard Lanoix, M.-L., Dimech, A., Dubuc, J., Poaty, B., Wu, R., McKenzie, J., Broda, S., and Chen, D. 2017. Controlling water infiltration in waste rock piles: Design, construction, and monitoring of a large-scale in-situ pilot test pile. GeoOttawa 2017.

Mine Environment Neutral Drainage Program (MEND). 1996. Évaluation en laboratoire de barrières sèches construites à partir de résidus miniers. Report 2.22.2a, Énergie, Mines et Ressources Canada. 
Morel-Seytoux, H. J. 1992. The capillary barrier effect at the interface of two soil layers with some contrast in properties. HYDROWAR Report 92.4 Hydrology Days Publications.

Morin, K. A., Gerencher, E., Jones; C. E., and Konasewich, D. E. 1991. Critical literature review of acid drainage from waste rock, MEND Report 1.11.1.

Newman, L. L., Herasymiuk, G.M., Barbour, S. L., Fredlund, D. G., and Smith, T. 1997. The hydrogeology of waste rock dumps and a mechanism for unsaturated preferential flow, in Proceedings ICARD 1997, pp. 551-564.

Nicholson, R. V. ,Gillham, R. W., Cherry, J. A., and Reardon, E. J. 1990. Reduction of acid generation in mine tailings through the use of moisture-retaining cover layers as oxygen barriers: Reply. Canadian Geotechnical Journal, 27(3), 402-403. doi:10.1139/t90-052.

Peregoedova, A., Aubertin, M., and Bussière, B. 2013. Laboratory measurement and prediction of the saturated hydraulic conductivity of mine waste rock. In GeoMontreal 2013: Geosciences for Sustainability, 66th CGS Conference, Montréal, Que.

Perzlmaier, S., Aufleger, M., and Conrad, M. 2004. Distributed fibre optic temperature measurements in hydraulic engineering - Prospects of the heat-up method, in Proceedings of the 72nd Annual Meeting-Workshop on Dam Safety Problems and Solutions-Sharing Experience, p. 31, Korean Natl. Comm. on Large Dams, Seoul.

Rasmusson, A., and Erikson, J. C. 1986. Capillary Barriers in covers for mine tailing dumps. Report 3307, The National Swedish Environmental Protection Board.

Sayde, C., Gregory, C., Gil-Rodriguez, M., Tufillaro, N., Tyler, S., Giesen, N. V., and Selker, J. S. 2010. Feasibility of soil moisture monitoring with heated fibre optics. Water Resources Research, 46(6). doi:10.1029/2009wr007846.

Sayde, C., Buelga, J. B., Rodriguez-Sinobas, L., Khoury, L. E., English, M., Giesen, N. V., and Selker, J. S. 2014. Mapping variability of soil water content and flux across 1-1000 m scales using the Actively Heated Fibre Optic method. Water Resources Research, 50(9), 7302-7317. doi:10.1002/2013wr014983.

Selker, J., Giesen, N. V., Westhoff, M., Luxemburg, W., and Parlange, M. B. 2006. Fibre optics opens window on stream dynamics. Geophysical Research Letters, 33(24). doi:10.1029/2006gl027979.

Sourbeer, J. J., and Loheide, S. P. 2015. Obstacles to long-term soil moisture monitoring with heated distributed temperature sensing. Hydrological Processes, 30(7), 1017-1035. doi:10.1002/hyp.10615

Striegl, A. M. and Loheide, S. P. 2012. Heated Distributed Temperature Sensing for Field Scale Soil Moisture Monitoring. Ground Water, 50: 340-347. doi:10.1111/j.1745-6584.2012.00928

Topp, G. C. Davis, J. L., and Annan, A. P. 1980. Electromagnetic determination of soil water content: Measurements in coaxial transmission lines, Water Resour. Res. 16(3), 574-582, doi:10.1029/WR016i003p00574.

Weiss, J. D. 2003. Using Fibre Optics to Detect Moisture Intrusion into a Landfill Cap Consisting of a Vegetative Soil Barrier. Journal of the Air and Waste Management Association, 53(9), 1130-1148. doi:10.1080/10473289.2003.10466268.

Wilson, J. A., Wilson, G. W., and Fredlund, D. G. 2000. Numerical modeling of vertical and inclined waste rock layers, in Proceedings of 5th International Conference on Acid Rock Drainage. Denver, Colorado, pp. 257-266.

Zubelzu, S., Rodriguez-Sinobas, L., Saa-Requejo, A., Benitez, J., and Tarquis, A. M. 2019. Assessing soil water content variability through active heat distributed fiber optic temperature sensing. Agricultural Water Management, 212, 193-202. doi:10.1016/j.agwat.2018.08.008 


\section{Figure Captions}

Figure 1: Column helices exposed in the upper sand layer, prior to emplacement of remaining sand layer. Two adjacent fibre optic cables are tied together $1 \mathrm{~cm}$ apart; however, only one cable is used for the experiment.

Figure 2: Fibre optic cable path (dotted lines continue into helical structures). The red dotted line is the heated portion of the fibre optic cable through which $15 \mathrm{~W} / \mathrm{m}$ of power was applied.

Figure 3: Experimental column containing fine sand (bottom left) and coarse screened anorthosite (bottom right).

Figure 4: a) Temperature profile along depth of column, as measured in the outer helix through time. Rain infiltration starts at 15:00. Change in temperature due to heating for b) dry, c) wet, and d) saturated conditions in the sand and anorthosite, measured in the outer helix. (Note saturated conditions are not represented in Figure 4a).

Figure 5: Volumetric water content calculated through time from the FO-DTS set up in the outer $(35 \mathrm{~cm}$ radius, 2 $\mathrm{cm}$ resolution), middle ( $25 \mathrm{~cm}$ radius, $3 \mathrm{~cm}$ resolution), and interior helix (15 $\mathrm{cm}$ radius helix, $5 \mathrm{~cm}$ resolution).

Figure 6: Scatterplot comparison of measured moisture, $\theta$, from FO-DTS and EC-5 $\theta$ in the sand (left) and anorthosite (right) layer of each helix.

Figure 7: 2D temperature representation of the column during dry conditions. The path of the three helices are outlined with their sampling points (dots and spatially averaged by $1 \mathrm{~m}$ ).

Figure 8: Depth vs. volumetric water content in each helix during dry (circle), wet (star), and saturated (triangle) conditions. 


\section{Figures}

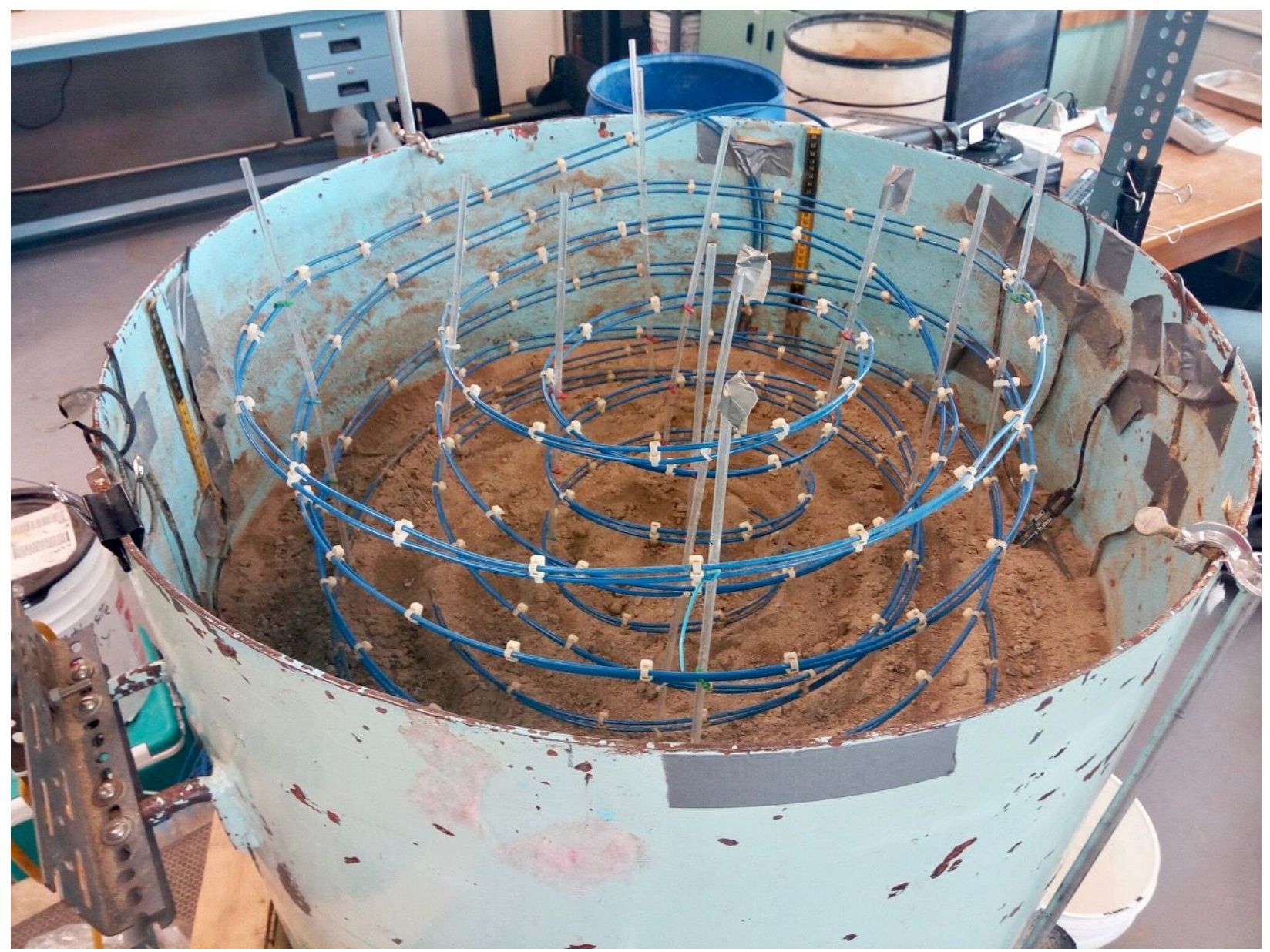

Figure 1: Column helices exposed in the upper sand layer, prior to emblacement of remaining sand layer. Two adjacent fibre optic cables are tied together $1 \mathrm{~cm}$ apart; however, only one cable is used for the experiment. 


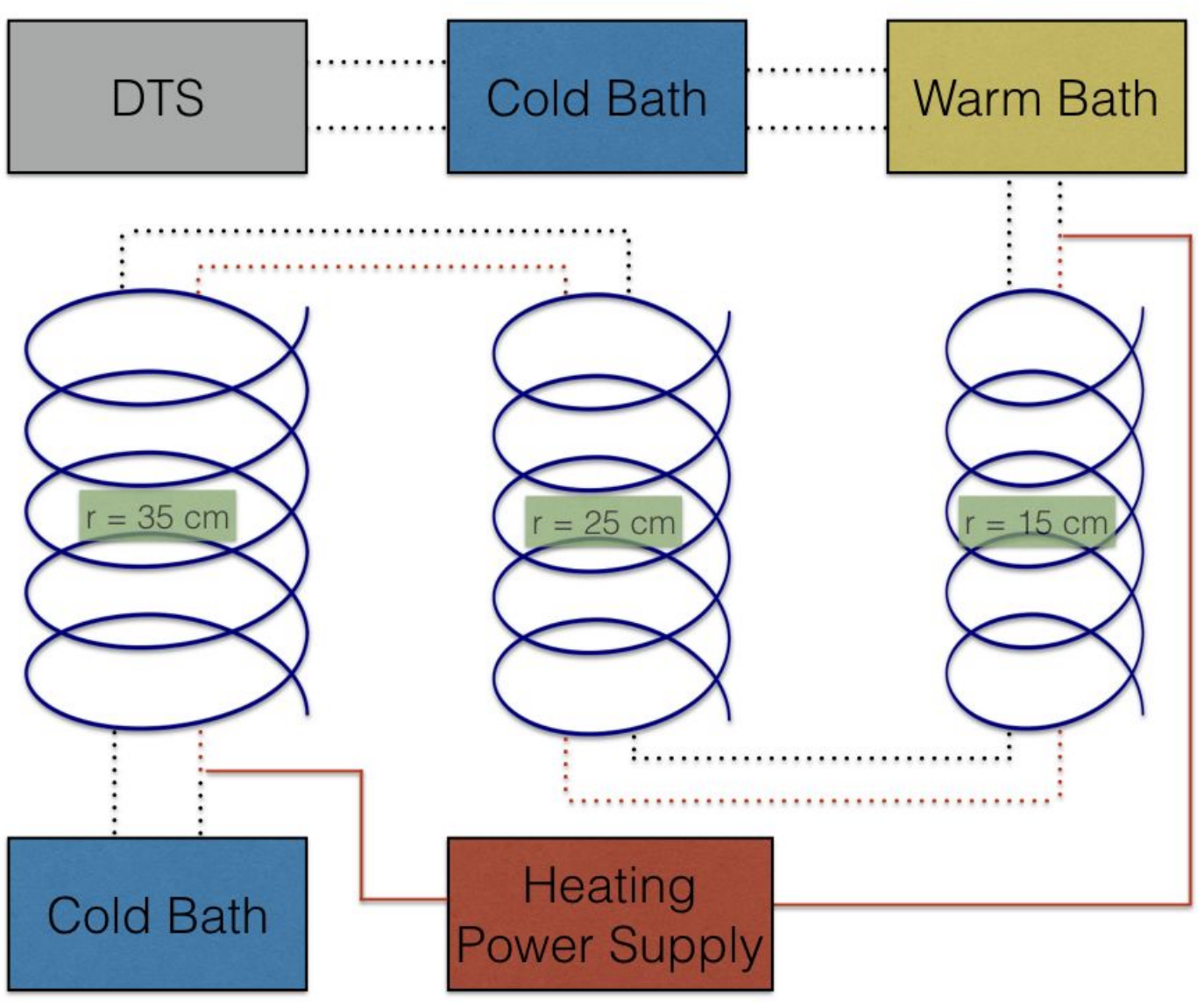

Figure 2: Fibre optic cable path (dotted lines continue into helical structures). The red dotted line is the heated portion of the fibre optic cable through which $15 \mathrm{~W} / \mathrm{m}$ of power was applied. 

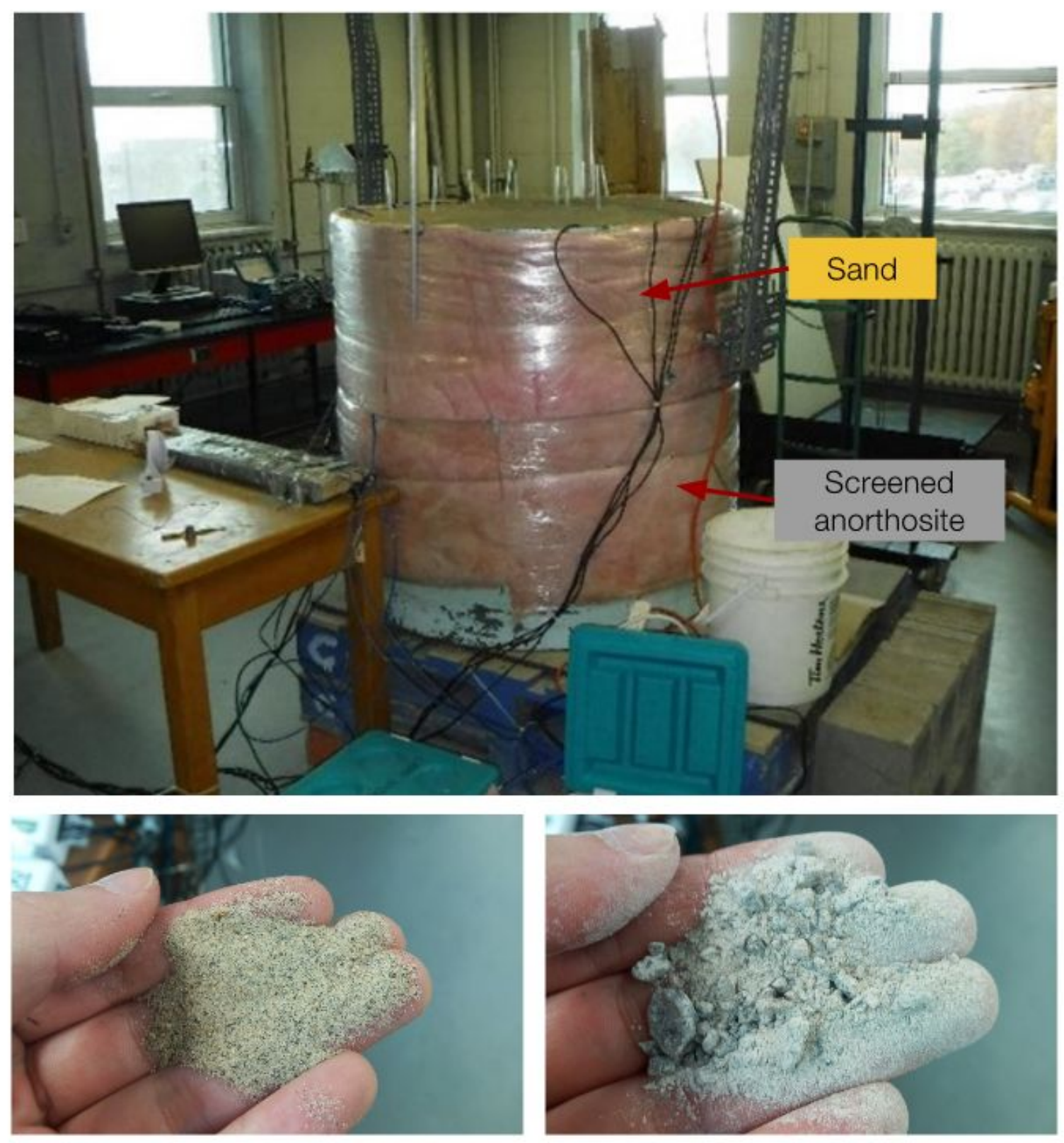

10 Figure 3: Experimental column containing fine sand (bottom left) and coarse screened anorthosite (bottom 11 right). 


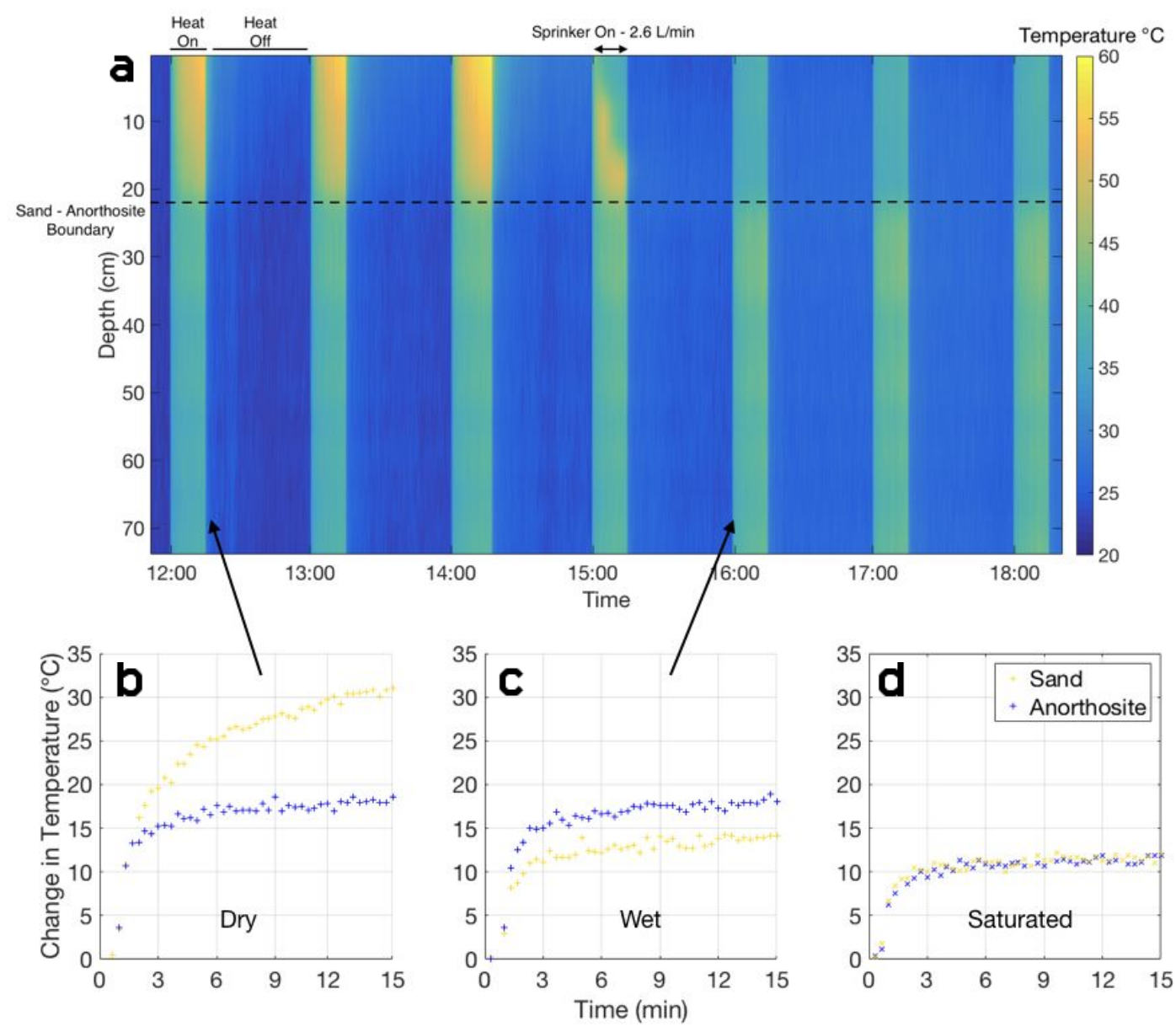

13 Figure 4: a) Temperature profile along depth of column, as measured in the outer helix through time. Rain 14 infiltration starts at 15:00. Change in temperature due to heating for b) dry, c) wet, and d) saturated conditions in the 15 sand and anorthosite, measured in the outer helix. (Note saturated conditions are not represented in Figure 4a). 


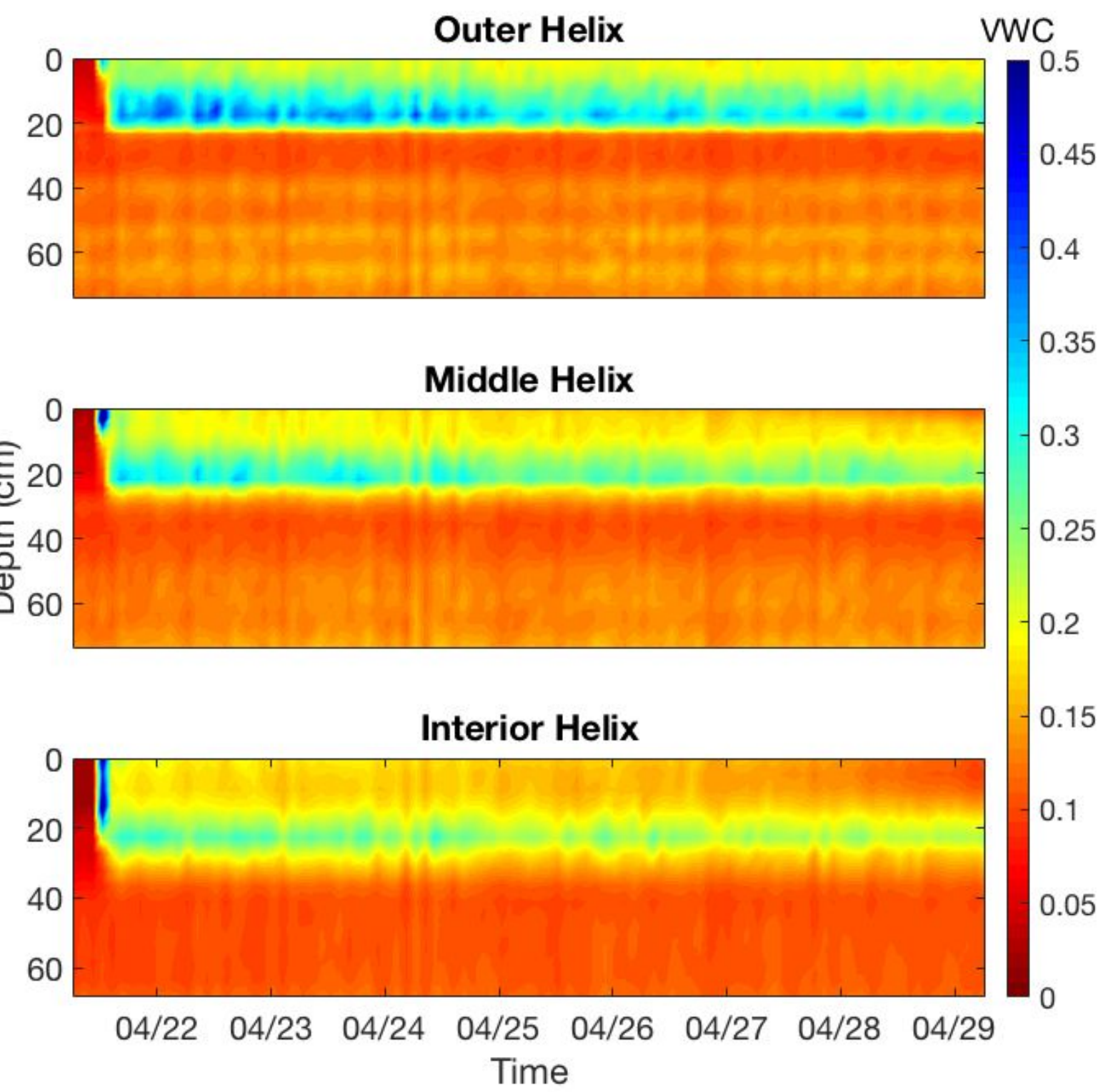

Figure 5: Volumetric water content calculated through time from the FO-DTS set up in the outer $(35 \mathrm{~cm}$ radius, 2 $\mathrm{cm}$ resolution), middle ( $25 \mathrm{~cm}$ radius, $3 \mathrm{~cm}$ resolution), and interior helix (15 $\mathrm{cm}$ radius helix, $5 \mathrm{~cm}$ resolution). 

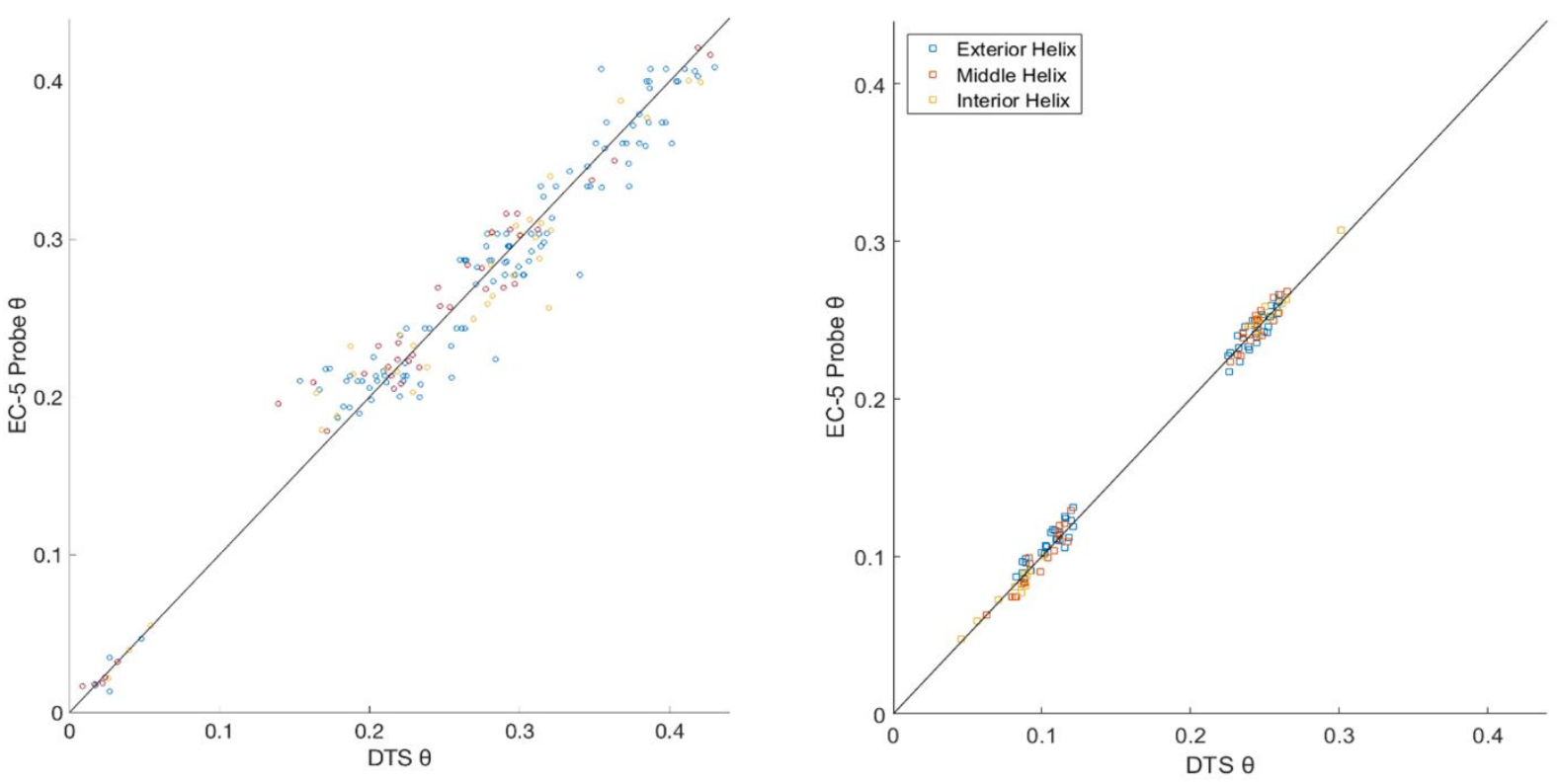

Figure 6: Scatterplot comparison of measured moisture, $\theta$, from FO-DTS and EC-5 $\theta$ in the sand (left) and 21 anorthosite (right) layer of each helix. 


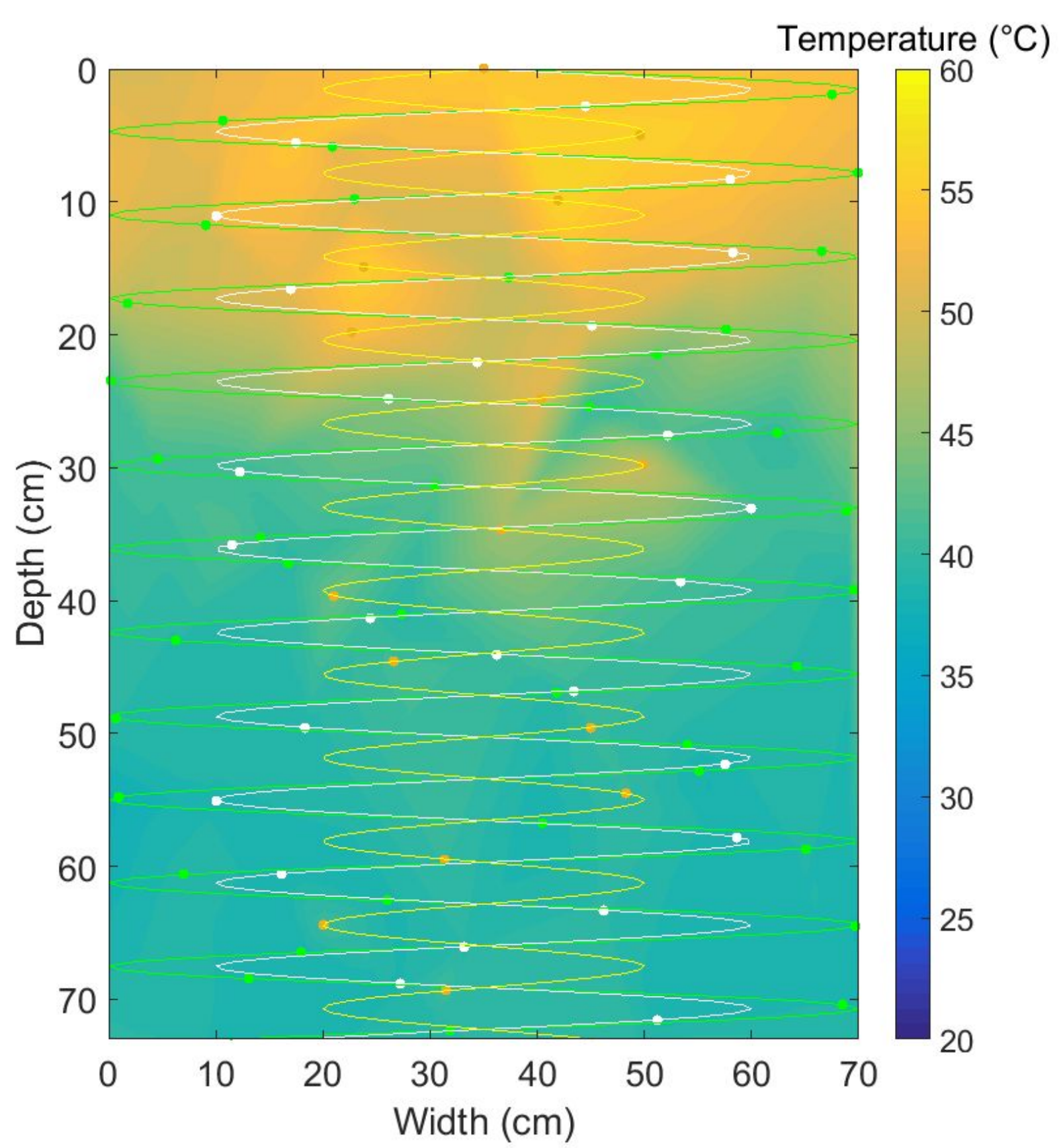

23 Figure 7: 2D temperature representation of the column during dry conditions. The path of the three helices are outlined with their sampling points (dots and spatially averaged by $1 \mathrm{~m}$ ). 


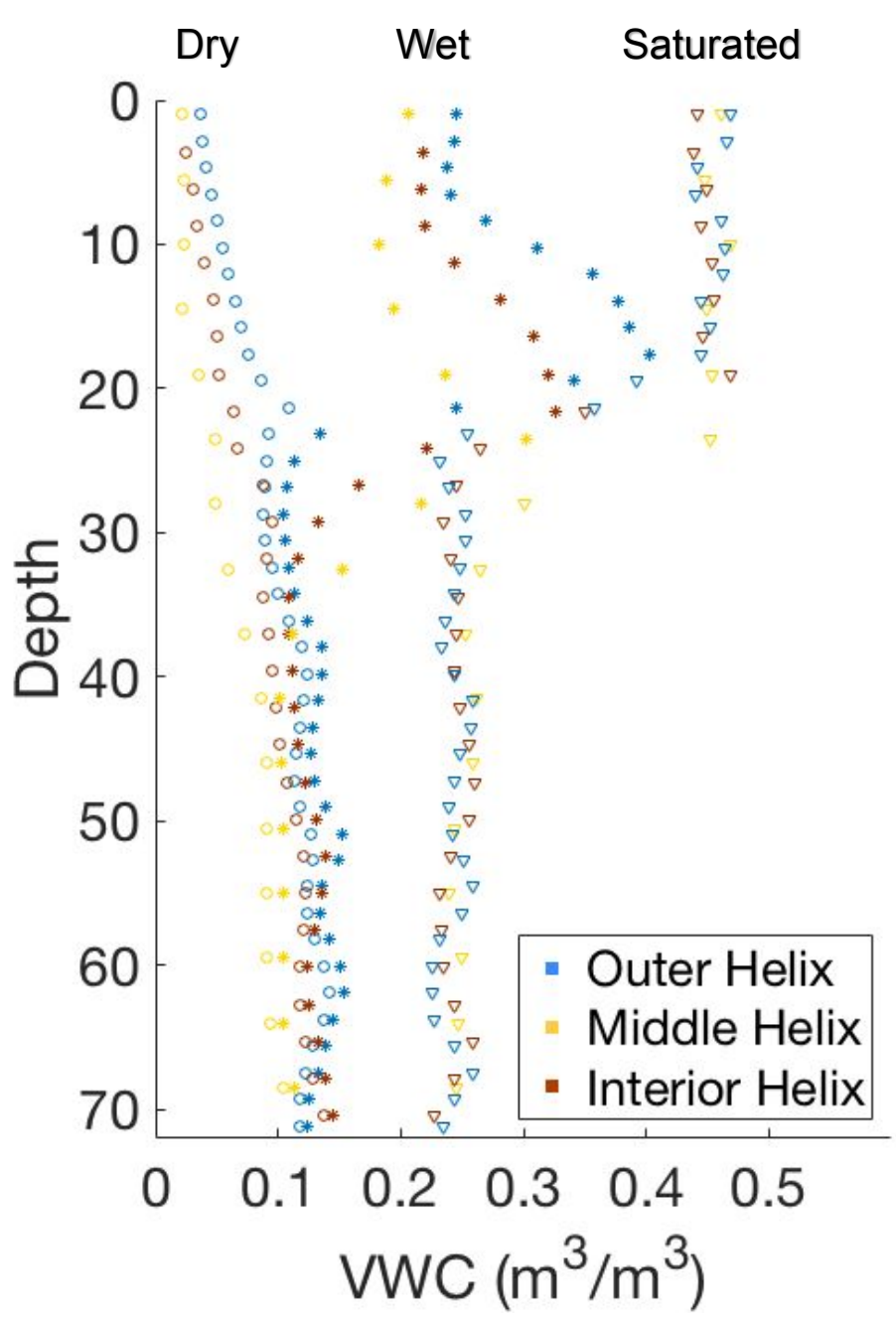

Figure 8: Depth vs. volumetric water content in each helix during dry (circle), wet (star), and saturated (triangle) conditions. 